\title{
A government-led effort to identify correlates of protection for COVID-19 vaccines
}

To the Editor-The unprecedented pace of vaccine development and deployment has been instrumental in controlling the COVID-19 pandemic, at least in high-income countries. Even in countries in which vaccination levels are high, several challenges remain, including demonstrating vaccine effectiveness in special populations (e.g., pediatric, pregnant and immunocompromised populations), determining the durability of vaccine-elicited immunity, and authorizing vaccines against viral variants and next-generation vaccines. Addressing these questions via separate, large efficacy studies in countries in which approved (or authorized) vaccines are available is ethically and logistically challenging.

Validation of a biomarker that is reliably predictive of vaccine efficacy, known as a 'correlate of protection' (CoP), may support approval of vaccines in lieu of large-scale efficacy studies ${ }^{1}$ (Fig. 1). To this end, the US government (USG) is coordinating an effort to identify such a CoP for COVID-19 vaccines.

Individual studies aimed at identifying antibody-based CoPs for COVID-19 vaccines are now emerging ${ }^{2}$. A correlation has been established between antibodies elicited by the ChAdOx1 nCoV-19 vaccine and protection ${ }^{3}$. The USG CoP effort is unique in encompassing the efficacy studies of five vaccines (comprising mRNA, adenoviral vector and recombinant protein platforms) and in its scale and subject diversity (over 120,000 participants with all major demographic groups well represented), as well as in the harmonization of its study clinical endpoints, immunoassays and statistical analysis plans ${ }^{4,5}$. Meta-analyses combining data across vaccine trials provide an unprecedented opportunity for assessing and validating CoPs across different populations, vaccine platforms and viral variants.

Conducting a CoP analysis for multiple large efficacy studies entails substantial operational complexity. Making efficacy results available early enough to inform clinical development amid an ongoing pandemic necessitates planning and coordination among the vaccine manufacturers conducting the clinical trials, the scientists developing the assays, the labs testing the samples and the statisticians conducting the analyses. These stakeholders have collaborated closely for the USG CoP effort, with the USG coordinating these various activities (Fig. 2).

The USG convened a team of leading virologists, immunologists, statisticians and clinical trialists to articulate the scientific and programmatic goals of the CoP effort, harmonize the study designs and implement a rigorous sampling, testing and analysis plan.

To measure antibody markers in participants in phase 3 trials, the USG opted to use a case-cohort-sampling design. This sampling approach was chosen for its operational flexibility, as it allows participants to be selected as soon as trial enrollment is complete. Stratification of this random sample ensured that vaccine immunogenicity could be characterized across different covariates (e.g., age and demographic factors, treatment status, and prior SARS-CoV-2 infection status). For each study endpoint-asymptomatic infection, mild to moderate or severe disease, and viral load at COVID-19 diagnosis-antibody markers were measured in all vaccine-breakthrough cases and the randomly sampled sub-cohort, totaling approximately $\sim 1,200$ vaccine recipients across all demographic and risk groups. The latter provided key antibody-marker data from noncase-control participants ${ }^{6}$.

The USG then partnered with academic and industry labs to develop, qualify and validate immunoassays that measure antibodies elicited by COVID-19 vaccines. Assays for quantifying antibodies that neutralize the infectivity of SARS-CoV-2 or bind to the viral spike protein were selected because they previously yielded CoP markers for other vaccines ${ }^{1,7}$. The USG chose the following assays for development and validation: electrochemiluminescence immunoassays that detect the binding of IgG antibody to the ectodomain or receptor-binding domain of spike protein; lentivirus pseudotype-neutralizing antibody assay (pseudovirus); and live virus-neutralizing antibody assay. As the assays advanced through development, qualification and validation plans were reviewed by the US Food and Drug Administration.

The USG accelerated assay development by securing priority access to reagents, facilitating information sharing between

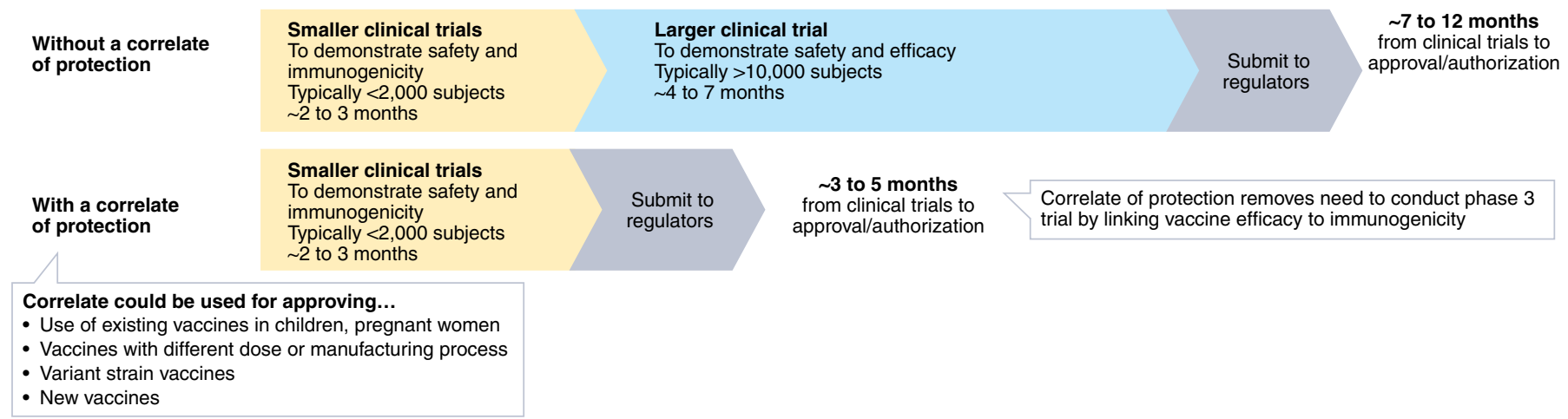

Fig. 1 | The impact of correlates of protection on vaccine clinical development. How timelines for vaccine development can be shortened with a known CoP. Yellow, small safety and immunogenicity trials; blue, large efficacy trials. 


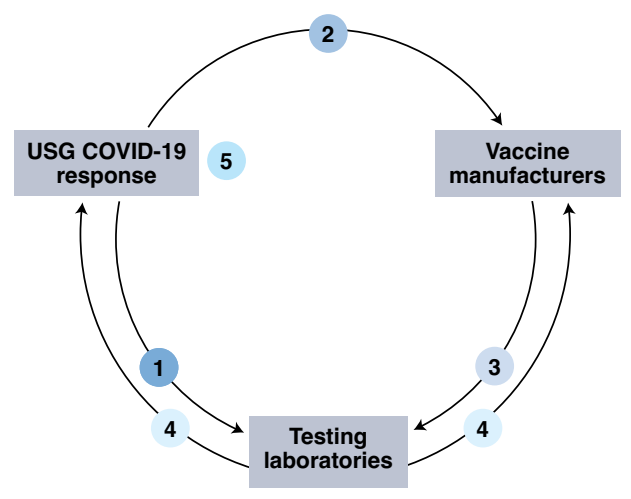

USG works with leading academic and

government laboratories to develop, qualify and

validate assays for testing company samples

USG develops statistical plan for conducting

2.

correlates of protection analysis, coordinates

sample selection with vaccine manufacturers

3

Vaccine manufacturers transfer samples for

testing in laboratories selected by USG

Laboratories test samples and share

immunogenicity data

USG conducts correlates of protection analysis

and shares results with the companies and the

broader scientific community

Fig. 2 | The role of stakeholders in USG correlates of protection analysis. Flow of information and coordination among the USG COVID-19 response team, vaccine manufacturers and testing laboratories in the process of generating CoPs.

labs, and engaging with regulators to ensure adherence to their specifications. Once an assay had progressed sufficiently, the USG coordinated its technology transfer from the developing lab to contract research organizations with greater lab testing capacity. The USG maintained a portfolio of assays to provide redundancy against assay failure and implemented an equivalency program to ensure assay concordance across testing labs.

The USG then worked closely with vaccine manufacturers to implement the CoP analysis within the context of their clinical trials. Serum samples from participants who acquired SARS-CoV-2 infection (with researchers blinded to sample identity), and from a random sample, as specified in the sampling plan, were identified, collected and tested in the immunoassays described above. To efficiently test tens of thousands of samples in a short period of time, the USG took an active approach to managing sample flow, matching vaccine manufacturers to particular testing labs on the basis of the timing of key trial milestones, sample availability and lab readiness/testing capacity.

As batches of samples were tested, the labs continuously transferred immunogenicity data to vaccine manufacturers. These data were then associated with the appropriate demographic/clinical endpoint data and were provided to the USG's statistical analysis team, which conducted an immunogenicity analysis and antibody-marker CoP analysis against the study endpoints in accordance with the analysis plan.

Finally, the USG, study sponsors and academic partners evaluated the results with the goal of making them available to the broader medical community via publication in peer-reviewed journals.

Our experience could serve as a model for similar efforts, in the context of COVID-19 or other public-health challenges, and highlights how a public body can provide the platform for manufacturers and academic scientists to collaborate and propel the science forward, without compromising healthy competition within the private sector. We are also excited for the potential use of these USG-developed assays for fulfilling clinical development priorities beyond identifying CoPs, such as for vaccines for tackling viral variants, and booster studies. We look forward to sharing the results of this unprecedented collaboration over the coming months.

\section{Richard A. Koup ${ }^{1,5}$,}

Ruben O. Donis $2,5 \bowtie$, Peter B. Gilbert ${ }^{3}$, Andrew W. Lii ${ }^{4}$ Najaf A. Shah ${ }^{4}$ and Christopher R. Houchens ${ }^{2}$

${ }^{1}$ Vaccine Research Center, National Institutes of Health, Bethesda, MD, USA. ${ }^{2}$ Biomedical Advanced Research and Development Authority, US Department of Health and Human Services, Office of the Assistant Secretary for Preparedness and Response, Washington, DC, USA.
${ }^{3}$ Fred Hutchinson Cancer Research Center, University of Washington, Seattle, WA, USA. ${ }^{4}$ Boston Consulting Group, Summit, NJ, USA. ${ }^{5}$ These authors contributed equally: Richard A. Koup, Ruben O. Donis.

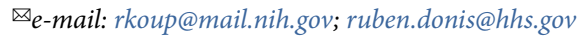

Published online: 13 September 2021 https://doi.org/10.1038/s41591-021-01484-6

References

1. Plotkin, S. A. Clin. Vaccine Immunol. 17, 1055-1065 (2010).

2. Khoury, D. S. et al. Nat. Med. 27, 1205-1211 (2021).

3. Feng, S. et al. Preprint at medRxiv https://doi. org/10.1101/2021.06.21.21258528 (2021).

4. Corey, L., Mascola, J. R., Fauci, A. S. \& Collins, F. S. Science 368, 948-950 (2020)

5. Slaoui, M. \& Hepburn, M. N. Engl. J. Med. 383, 1701-1703 (2020).

6. USG COVID-19 Response Team/Coronavirus Prevention Network (CoVPN) Biostatistics Team. figshare https://doi. org/10.6084/m9.figshare.13198595.v12 (2021).

7. Haynes, B. F. et al. N. Engl. J. Med. 366, 1275-1286 (2012).

\section{Acknowledgements}

We thank the following people for their important contributions and input: M. Hepburn, R. Johnson, J. Mascola, D. Montefiori, A. McDermott, T. Denny, J. Lathey, M. Marovich, M. Robb, P. D’Souza, K. Martins, F. Castellino, L. Jayashankar, C. Hoffman, L. Watson, E. Sturtevant, C. Ventura, D. Follmann, A. Randhawa, J. Zhou, Y. Fong, X. Tong, T. Faison, D. Turley, N. El-Badry, M. Del Vecchio, N. Mytle, D. Benkeser, T. Hu, C. Badorrek and A. Kolhekar. The content and opinions in this article reflect solely the views of the authors, and do not represent those of the US government.

Author contributions

All authors contributed to the writing and editing of the manuscript.

Competing interests

The authors declare no competing interests. 